\title{
Electroretinographic modifications induced by agomelatine: a novel avenue to the understanding of the claimed antidepressant effect of the drug?
}

This article was published in the following Dove Press journal:

Neuropsychiatric Disease and Treatment

20 May 2014

Number of times this article has been viewed

\author{
Michele Fornaro' \\ Fabio Bandini ${ }^{2}$ \\ Luca Cestari ${ }^{3}$ \\ Christian Cordano 4 \\ Carla Ogliastro ${ }^{4}$ \\ Claudio Albano 4 \\ Domenico De Berardis 5 \\ Matteo Martino 6 \\ Andrea Escelsior ${ }^{6}$ \\ Giulio Rocchi ${ }^{6}$ \\ Pantaleo Fornaro ${ }^{6}$ \\ Concetta De Pasquale' \\ 'Department of Educative Science, \\ University of Catania, Catania, Italy; \\ ${ }^{2}$ Department of Neurology, San Paolo \\ Hospital, Savona, Italy; ${ }^{3}$ Department \\ of Ophthalmology, University of \\ Pisa, Pisa, Italy; ${ }^{4}$ Department of \\ Neurosciences, Ophthalmology \\ and Genetics - Section of Neurology, \\ University of Genoa, Genoa, Italy; \\ ${ }^{5}$ National Health Service, Department \\ of Mental Health, Psychiatric Service \\ of Diagnosis and Treatment, Hospital \\ G. Mazzini, ASL 4, Teramo, Italy; \\ ${ }^{6}$ Department of Neurosciences, \\ Ophthalmology and Genetics - \\ Section of Psychiatry, University \\ of Genoa, Genoa, Italy
}

Correspondence: Michele Fornaro Department of Educative Science, University of Catania,

Via Teatro Greco, 84, ZIP 95 I25,

Catania, Italy

Tel +393474l 40003

Fax +39010 3537669

Email dott.fornaro@gmail.com
Background: Agomelatine, the first melatonergic antidepressant, has been postulated to enhance the dopaminergic activity at the central nervous system by 5-hydroxytryptamine receptor type $2 \mathrm{C}$ (5-HT2C) antagonism, yet the impact of melatonergic agonism on this pathway is unclear. Previous studies employing simplified, yet reliable, proxy (retinal) measures of the central nervous system dopaminergic activity, namely the standard electroretinogram (ERG) technique, suggested a reduction of the dopaminergic activity of the main ERG parameter, the b-wave, by pure melatonin, notably a hormone devoid of any antidepressant activity. Therefore, the antidepressant effects of the melatonergic antidepressant drug agomelatine should be reflected by a differential b-wave trend at ERG versus the effect exerted by pure melatonin, which was eventually found to be due to a contrasting effect on central dopaminergic transmission between the two drugs.

Objective and methods: The aim of the present preliminary ERG study carried out on healthy volunteers $(n=23)$ receiving agomelatine was to explore the impact of this antidepressant drug on b-wave amplitude and latency of cones in daylight conditions using standard ERG.

Results: As postulated, agomelatine induced an enhancement of retinal dopaminergic activity, in contrast to what has been previously documented for melatonin.

Conclusion: Given the limits of this explorative study, especially the lack of a control group and that of a luminance response function to measure retinal sensitivity, further studies in clinical samples are recommended to allow more tenable conclusions about the potential role of ERG in discriminating between 5-HT antagonism and melatonergic (MT) agonism in relationship to the claimed antidepressant effect of agomelatine.

Keywords: electroretinogram, ERG, dopamine, 5-HT2C

\section{Introduction}

Currently, virtually any psychiatric diagnosis is made through a combination of patient interviews, checklists, or self-report questionnaires, which rely on the symptoms coded in the Diagnostic and Statistical Manual of Mental Disorders, either the fourth edition ${ }^{1}$ or the recently introduced fifth edition. ${ }^{2}$ Yet, considerable debate exists about the actual validity of this sole symptom-based approach, thus sustaining the interest in objective biomarkers toward a better understanding of psychiatric disorders, including major depressive disorder (MDD), and their pharmacological response. ${ }^{3}$ Furthermore, a number of issues, including heterogeneous patients' variables, high medical and psychiatric comorbidity rates, medications/triggers, inconsistency in specimen collection, storage and measurement protocols, and complexity of neuropsychiatric biological determinants of disorders, hinder the search for reliable biomarkers. ${ }^{4,5}$ Ideally, a hassle-free biomarker reflecting the 
central nervous system (CNS) functioning should provide a simplified, yet reliable, proxy measure of the activity of those neurotransmitters that are supposed to be involved in major psychiatric disturbances, to be flexibly applied both to clinical and healthy samples. Among other approaches, the electroretinogram (ERG) represents a relatively noninvasive, short, and cost-effective method developed to investigate the origin of a visual loss due to a retinal disease or injury, possibly representing a proxy of global CNS activity of dopamine, ${ }^{6}$ a core monoamine in depression, directly or indirectly modulated by a number of antidepressant drugs with different pharmacodynamics. ${ }^{7}$ Specifically, since the retina is part of the CNS due to its embryonic origin, the ERG has been used to investigate not only MDD, but also seasonal affective disorder, schizophrenia and bipolar disorder, autism spectrum disorders, and drug addiction, ${ }^{8}$ both in clinical and healthy samples exposed to different pharmacological agents, ${ }^{8-17}$ as well as in animal samples. ${ }^{6,18}$ Of note, dopamine modulates different retinal functions, including counterbalancing of the synthesis of melatonin as measured with the ERG. ${ }^{19-21}$ Melatonergic activity is also often impaired in the course of depressive episodes with prominent circadian rhythm disturbances, even if there is no antidepressant effect firmly documented for the hormone melatonin, neither for its analogue, ramelteon. ${ }^{22-25}$ This seems quite puzzling considering that the recently released pro-melatonergic antidepressant agomelatine has been approved in Europe for the treatment of MDD, though not in the US. ${ }^{26,27}$ A potential explanation for the claimed antidepressant effect of agomelatine should nonetheless be represented by its 5-hydroxytryptamine receptor type 2B (5-HT2B) and 5-HT2C serotonergic antagonism, ${ }^{28}$ which may also promote the dopaminergic firing at the ventral tegmental area, frontal cortex, hypothalamus, hippocampus, medulla and pons, and also the retina, which is part of the CNS as well, ${ }^{29}$ via enhancement of norepinephrinergic activity at the locus coeruleus. ${ }^{30}$ Yet, while there is no question about the impact of melatonergic modulation on circadian rhythms relevant for mood as well as other core functions, the discrimination of the most plausible antidepressant effect of agomelatine between 5-HT antagonism versus melatonergic receptor type 1 (MT1) and type 2 (MT2) agonism remains elusive, at least with regard to just the simplified paradigm of dopaminergic modulation. Additionally, agomelatine has been proposed to indirectly modulate even the release of glutamate from prefrontal and frontal cortex and hippocampus, ${ }^{31,32}$ which further hinders the identification of a sole putative neurobiological pathway accounting for the claimed antidepressant effect of the drug.
Therefore, the aim of this preliminary study carried out on healthy volunteers was to assess the impact of agomelatine on retinal dopaminergic activity measured using standard ERG recording, to provide a dissertation on the potential clinical implications of a differential neuropsychopharmacological ERG trend eventually observed for agomelatine versus the trend already documented for melatonin.

\section{Methods}

\section{The flash ERG: essential} neuropsychopharmacological foundations

The light reaches the photoreceptors (either cones or rods) hosted at the outer segment of the retina after crossing the anterior segment of the eye, then is absorbed by the photopigment of the photoreceptors, which ignites the phototransduction process. In dark conditions, photoreceptors rest in a depolarized state, while the photon absorption leads to their hyperpolarization followed by the depolarization of the ON bipolar cells. Subsequently, the electric signal spreads to the ganglion cells, the axons of which reach the brain, mainly converging at the visual cortex. The retina also includes horizontal cells and amacrine cells, which are interneurons joining photoreceptors and bipolar cells, as well as the Müller cells acting as glia. ${ }^{8}$

Since dopamine represents a core retinal neurotransmitter involved in signal transduction, the ERG should be a convenient technique by which to assess such dopaminergic activity both in light- and dark-adapted conditions, ${ }^{33}$ essentially by measurement of the "implicit time" and "amplitude" parameters of the b-wave, ${ }^{34}$ a trace component of the ERG primarily determined by the bipolar cells. ${ }^{35-37}$ Any variation of the intensity or chromatic characteristic of the light stimulus could also be assessed by the ERG, either for rods, cones, or mixed rod/cone photoreceptors functioning, thus allowing an objective quantitative measure of the retinal sensitivity to light. ${ }^{34}$ Dopamine released by the amacrine and interplexiform cells ${ }^{38}$ interacts with the D1-like receptors (namely, D1 and D5 subtypes) at the horizontal, bipolar, amacrine, and ganglion cells, and with the D2-like (D2, D3, and D4) receptors located at the retinal pigment epithelium cells, photoreceptors, and Müller glial cells, ${ }^{39-43}$ all of which are involved in the modulation of a number of retinal functions, including melatonin release. ${ }^{20}$ Also, the daily synthesis and release of retinal dopamine, which is primarily influenced by lighting condition, follows a 24 -hour rhythm, ${ }^{44}$ being influenced by the interaction between the amacrine and interplexiform dopaminergic neurons and photoreceptors. Enhancement of dopamine activity via activation of D2-like receptors (namely D4 ones) located on photoreceptors inhibits the synthesis of melatonin, leading to subsequent inhibition of 
serotonergic $\mathrm{N}$-acetyltransferase activity. ${ }^{45,46}$ In mammals, the D4 receptor accounts for the effects of the ligands on the whole D2 receptor family, ${ }^{42}$ either at the rods, cones, or retinal pigment epithelium, ${ }^{47}$ which, in turn, ultimately account for the impact of dopamine in the regulation of melatonin biosynthesis in vertebrate retina. ${ }^{48}$ Substance $\mathrm{P}$ and dynorphin, which are expressed in D1 receptor-containing neurons, as well as preproenkephalin in D2 receptor-containing neurons, have also been used as monitors of dopaminergic activity in the $\mathrm{CNS},{ }^{49}$ whereas there have been conflicting reports as to whether D1-like receptors are capable of increasing or decreasing the potassium efflux: D1-like agonists increase potassium current from chick retinal cells via an adenosine monophosphate (AMP)-independent mechanism, but inhibit this efflux in rat striatal neurons, ${ }^{50}$ as such, a conclusive understanding of the actual effect of D1 (and possibly D5) stimulation or inhibition at the retina likewise remains elusive. ${ }^{51}$ Melatonin suppresses the retinal release of dopamine via activation of its own melatonergic receptors (primarily MT1, but also MT2 and MT3 subtypes), widely distributed in the $\mathrm{CNS},{ }^{52}$ including the retinal amacrine and interplexiform dopaminergic neurons. ${ }^{53,54}$

As mentioned previously, the b-wave response of the ERG is influenced both by dopaminergic and melatonergic modulation. Specifically, in daylight conditions, the b-wave response seems linked to cone dominance, possibly related to a shift toward a relative dominance of dopaminergic tone. On the contrary, under scotopic conditions, the b-wave response could be influenced by a relative dominance of rod tone,${ }^{55}$ when the presence of circulating melatonin could account for rod dominance. ${ }^{56}$

ERG studies carried out on healthy volunteers receiving melatonin, performed during conditions usually characterized by the absence of the hormone itself, showed a decline of cone response, either recorded as a decrease of the b-wave amplitude at $10 \mathrm{mg} /$ day, ${ }^{57}$ or as a significant reduction of cones' ERG maximal response associated to a prolonged implicit time with $15 \mathrm{mg} /$ day melatonin. ${ }^{58}$ Concordant results were recently documented in animal samples exposed to high doses of melatonin during the day $(90 \mathrm{mg} /$ day in anaesthetized beagle dogs), showing a decrease of the photopic amplitude of both a- and b-waves, but no impact on the implicit time, which may indicate that the negative impact of melatonin on the photopic system may promote night vision. ${ }^{21}$

Agomelatine shares the MT1 and MT2 agonism provided by melatonin, but not the agonism on MT3 receptors, with MT3 receptors having not been documented in mammalian samples..$^{59}$ By blocking 5-HT2C receptors, agomelatine promotes the CNS release of dopamine, possibly at the retina as well. However, at the time of writing, there are no available data whatsoever about the investigation of agomelatine using the flash ERG in contrast to previous evidence for melatonin, this being the aim of this preliminary study.

\section{Study subjects}

Twenty-three healthy volunteers, both sexes, were enrolled at the Department of Neuroscience of the San Martino Hospital of Genoa, Genoa, Italy, between September 2012 and March 2013, upon approval by the local ethical committee and patient signatures on a valid informed consent form after procedures had been fully explained by the investigators (either psychiatrists or neurologists). All procedures were carried out in accordance with the Declaration of Helsinki about human experimentation. ${ }^{60}$ No liver function monitoring was needed due to the prescription of just a single dose of agomelatine. In order to avoid any age-dependent effect affecting the $b$-wave amplitude, ${ }^{61}$ all subjects were aged between 22 and 35 years old. In addition, all subjects received the same single dose of agomelatine ( $25 \mathrm{mg}$ /day), avoiding any concomitant medication to limit potential confounding biases as much as possible. Similar considerations led to the inclusion of female subjects only during the luteal phase of the menstrual cycle (objective measure of luteinizing hormone levels: range 1-20 IU/L) in order to avoid an additional confounding factor potentially affecting the b-wave amplitude. ${ }^{62}$ Finally, the baseline ERG assessments (with each session requiring about 1 hour for completion) were performed according to the following scheme: 1) first ERG recording at 10 am (baseline $=\mathrm{T} 0$ ), immediately followed by oral intake of agomelatine $25 \mathrm{mg}$ (one pill); 2) 1 hour's rest; 3) second ERG recording; 4) 1 (more) hour's rest; 5) third (final) ERG measurement. Finally, a pictorial description of the standard ERG has been provided in Figure 1 along with a synthetic flow of study procedures in Figure 2.

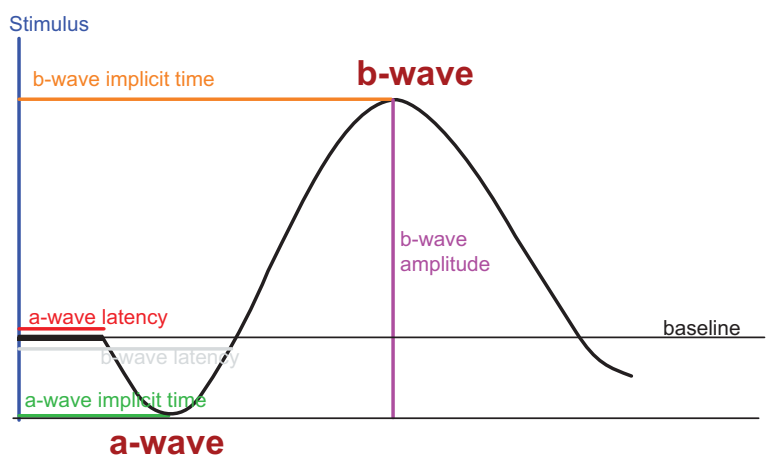

Figure I The a-wave of the electroretinogram reflects the activity of photoreceptors; the b-wave indicates the activity of the amacrine, horizontal, bipolar, and Müller cells. Implicit time refers to the time occurring between the initiation of the light stimulation (flash) and the peak of a- and b-waves. The a-wave amplitude was measured from baseline to a-wave trough; the b-wave amplitude was measured from a-wave trough to b-wave peak. 


\section{First ERG assessment (baseline)}

Enrolled healthy volunteers received their oral prescription of $25 \mathrm{mg}$ agomelatine (one pill) just following their first ERG assessment (which usually took about 1 hour, each session).

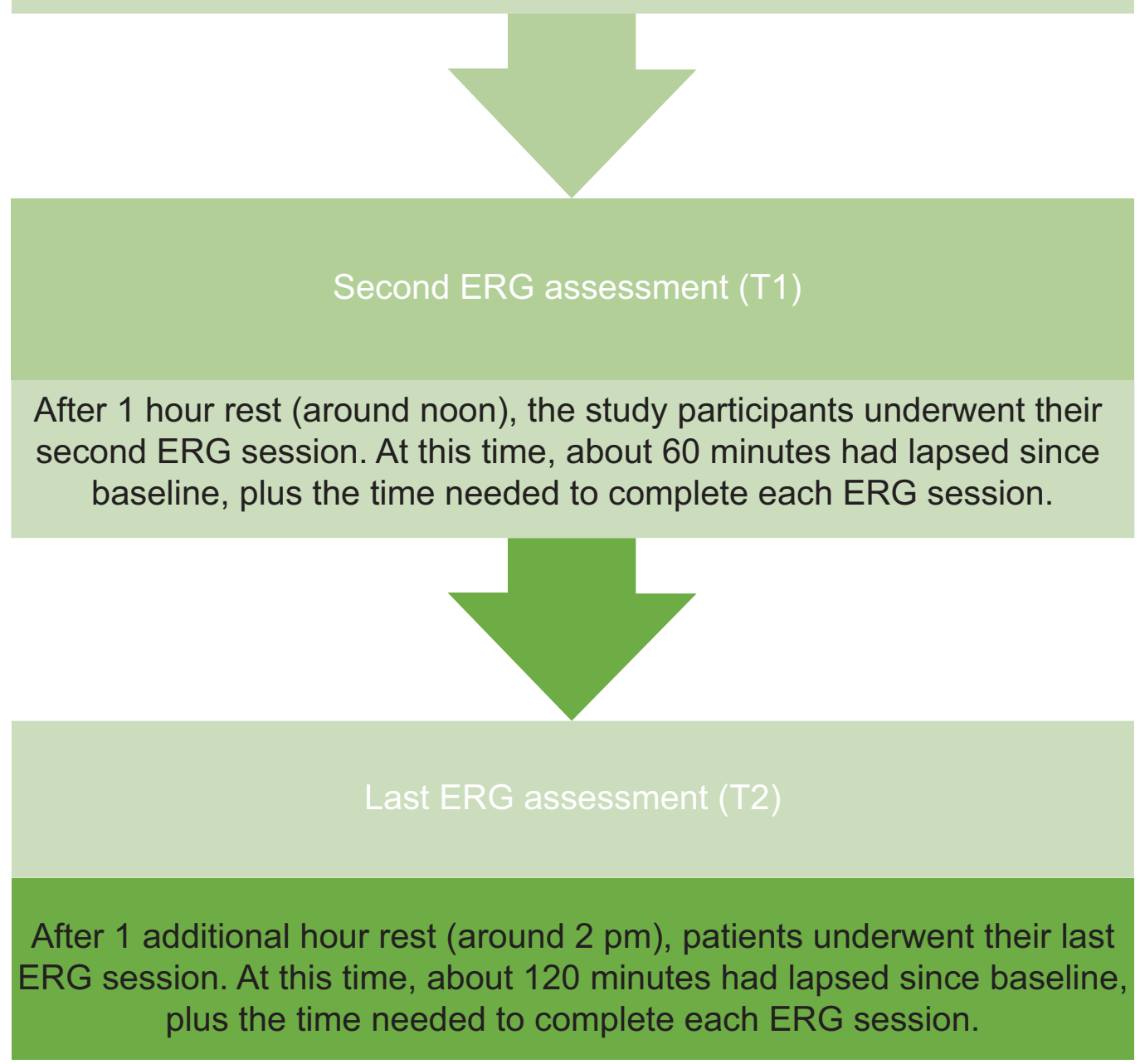

Figure 2 Flowchart of study procedures. Abbreviation: ERG, electroretinogram.

\section{Stimulation and recording}

All subjects underwent a full-field ERG recording. The electric signal was recorded binocularly (dilated pupils) using $9 \mathrm{~mm}$ silver/silver chloride skin disc electrodes placed on the lower eyelid; the reference electrodes were placed close to the outer canthus of the eye and the ground electrode was placed on the forehead. The electrode impedance was maintained below $5 \mathrm{k} \Omega$ in order to provide consistent results independent of the response yielded by skin electrodes with lower amplitude and higher noise levels with regard to either the corneal or conjunctival electrodes. The band-pass filter was set between $0.1 \mathrm{~Hz}$ and $500 \mathrm{~Hz}$, and the subjects were dark-adapted for 20 minutes before recording. A full-field Ganzfeld stimulator was used throughout the study, with the subjects seated in front of the stimulator bowl fixating on a point incorporated in the stimulus dome during each session. The dark-adapted 0.01 ERG (rod response) was recorded using a dim white flash of $0.01 \mathrm{cdsm} 2$. The darkadapted 3.0 ERG (combined rod-cone response) was generated by a white 3 cdsm 2 flash. After 10 minutes of light 
adaptation, the light-adapted 3.0 ERG (single-flash cone response) was recorded, using a 3 cdsm 2 stimulus with a background luminance of $30 \mathrm{~cd} / \mathrm{m} 2$ measured at the surface of the full-field stimulus bowl. b-wave amplitude and time to peak (implicit time) were measured (ms) for all ERGs. The a-wave was measured when detectable. The a-wave amplitude was measured from baseline to a-wave trough, while the b-wave amplitude was measured from a-wave trough to b-wave peak $(\mu \mathrm{V})$; the a-wave and b-wave implicit times were measured from the time of the flash to the peak of each wave.

\section{Statistical analysis}

Demographic and descriptive analyses were carried out using the IBM SPSS ${ }^{\circledR}$ software package (v 21) for Microsoft Windows $^{\circledR}$ (v 7) (IBM Corporation, Armonk, NY, USA). Since data follow a nonparametric distribution, as assessed by the Shapiro-Wilk test, Friedman's analysis of variance was used in order to compare the median scores of the ERG parameters.

\section{Results}

Twenty-three subjects (females $n=8$ or $35 \%$ of the sample; males $n=15$ or $65 \%$ ) were included in the study and completed all the scheduled appointments and procedures. The average age was $26.61 \pm 3.3$ years. Notably, among different measures of the b-wave parameters for both eyes (including latency and amplitude either for cones or rods, and for ERG maximal response), the only statistically significant modifications observed across the study were those related to cones' b-wave amplitude and latency, in both eyes (Table 1). Finally, no statistically significant difference was observed for such parameters in males versus females.

\section{Discussion}

\section{Limitations of the study}

The absence of a control group, including differential doses of agomelatine, placebo, or melatonin, or depressed controls, make the present results merely indicative at this time. Moreover, while dopamine could also affect the ERG, its effect was basically observed in terms of changes in the retinal sensitivity. ${ }^{63}$ Therefore, in this study, the lack of a luminance response function to measure retinal sensitivity with the ERG prompts for caution in the interpretation of our preliminary findings. Oscillatory potentials most likely generated by the dopaminergic neurons of the amacrine (or interplexiform) cells, should also be accounted for in replication studies. Similarly, we followed the International Society for Clinical Electrophysiology of Vision (ISCEV) Standard ${ }^{64}$ to record the ERG, which represents the minimum standard flashes to be used to investigate the retinal function (for patients with an eye disease); however, in terms of mere research purposes, assessing the dynamic of retinal functioning over a wide range of intensities should allow a more reliable assessment of the retinal sensitivity. Specifically, this latter consideration may have hampered the validity of the present preliminary protocol in the investigation of a possible impact of the ERG.

\section{Clinical and neuropsychopharmacological implications of study results}

In our sample, agomelatine induced a slight, yet statistically significant, increase of the cones' b-wave amplitude and latency in both eyes. While it may be argued that the above mentioned change in amplitude may actually be within the normal variation of the measure, this is nonetheless in contrast to previous reports ${ }^{56-58}$ from comparable ERG studies involving healthy subjects receiving melatonin. While both melatonin and agomelatine act on melatonergic receptors, the presence of 5-HT2C (and 5-HT2B) antagonism for the antidepressant drug should be prudently hypothesized to account for the differential trend observed at ERG, eventually observed to play a role in the therapeutic effect claimed in depressed samples. Notably, a previous polysomnographic study

Table I Slightly, yet statistically, significant trends of increase of b-wave parameters

\begin{tabular}{|c|c|c|c|c|c|c|}
\hline & \multicolumn{3}{|l|}{$\underline{\text { LE }}$} & \multicolumn{3}{|l|}{$\underline{\mathbf{R E}}$} \\
\hline & Baseline value & 60 minutes & 120 minutes & Baseline value & 60 minutes & 120 minutes \\
\hline \multicolumn{7}{|c|}{ Amplitude of the cones \pm SD } \\
\hline $\begin{array}{l}(\operatorname{LE} P=0.026 \\
\operatorname{RE} P=0.009)^{*}\end{array}$ & $49.22 \pm|8.7|$ & $52.30 \pm 18.43$ & $55.52 \pm 20.95$ & $45.35 \pm 18.48$ & $48.65 \pm 17.02$ & $51.56 \pm 17.22$ \\
\hline \multicolumn{7}{|c|}{ Cone latency $\pm S D$} \\
\hline $\begin{array}{l}(\operatorname{LE} P=0.00 \mathrm{I} ; \\
\operatorname{RE} P=0.00 \mathrm{I})^{*}\end{array}$ & $33.83 \pm 2.79$ & $34.74 \pm 3.67$ & $36.39 \pm 3.13$ & $33.52 \pm 2.69$ & $34.48 \pm 3.33$ & $36.17 \pm 3.08$ \\
\hline
\end{tabular}

Notes: Modifications that were not statistically significant are omitted (Friedman's two-way analysis of variance by ranks). REs and LEs were analyzed separately. Amplitude values are in $\mu \mathrm{V}$ (latency in $\mathrm{ms}$ ). All results were obtained in daylight conditions. *Provided $P$-values for the nonparametric test indicated to reject the null hypothesis (of a difference observed by chance); asymptotic significance not reported; significance level set to $P=0.05$.

Abbreviations: LE, left eye; RE, right eye; SD, standard deviation. 
carried out on a small sample of healthy volunteers with the same dose of agomelatine $(25 \mathrm{mg} /$ day) raised doubts about the actual blockade of 5-HT2C receptors in humans, based on the observation that this agent failed to increase slow-wave sleep, whereas potent 5-HT2C antagonists (eg, cyproheptadine $4 \mathrm{mg}$, ritanserin $5 \mathrm{mg}$, olanzapine $5 \mathrm{mg}$, or mirtazapine $30 \mathrm{mg}$ ) did. ${ }^{65}$ Furthermore, in a recent animal study, neither the long-term administration of melatonin ( $40 \mathrm{mg} / \mathrm{kg} /$ day) nor the selective $5-\mathrm{HT} 2 \mathrm{C}$ receptor antagonist SB $242084(0.5 \mathrm{mg} / \mathrm{kg} /$ day $)$ had an effect on the firing rate and burst parameters of serotonergic and dopaminergic neurons. The combination of these latter mechanisms, however, enhanced only the number of spontaneously active dopaminergic neurons at the ventral tegmental area, while leaving the firing of the serotonergic neurons unaltered at the raphe dorsal nucleus. Finally, the addition of a specific 5-HT2B antagonist (LY 266097 at $0.6 \mathrm{mg} / \mathrm{kg} / \mathrm{day}$ ), which proved by itself to be devoid of effect, to the previous dual regimen increased the number of bursts per minute of dopaminergic neurons and the percentage of spikes occurring in burst, which ultimately mimics the effect of the antidepressant agomelatine. ${ }^{66}$

\section{Conclusion}

Agomelatine may differ from melatonin not only in the absence of MT3 agonism, but also in the presence of a dual antagonism on 5-HT2C and 5-HT2B, which may corroborate the claimed clinical antidepressant effect and account for the increase of the b-wave amplitude and latency of the cones (in daylight conditions). Nonetheless, along with the abovementioned limitations urging much prudence in the interpretation of our results and the acknowledgement that a decline in the central serotonin activity may itself affect the cone b-wave latency, it pays to note that the "classical" paradigm of monoamines (including dopamine) as a core pathway involved in depression has been debated, shifting more attention toward impaired circadian rhythms in depression. ${ }^{67}$ Therefore, with the ultimate goal of shedding further light on the mechanism of action of novel antidepressants, with agomelatine being the case in point, additional, more methodologically accurate ERG studies on the matter are warranted.

\section{Acknowledgment}

We would like to thank all the healthy volunteers for their participation in this study.

\section{Disclosure}

The authors report no conflicts of interest in this work.

\section{References}

1. Diagnostic and Statistical Manual of Mental Disorders. 4th ed. Washington, DC: American Psychiatric Association; 1994.

2. Diagnostic and Statistical Manual of Mental Disorders. 5th ed. Washington, DC: American Psychiatric Association; 2013.

3. Lopresti AL, Maker GL, Hood SD, Drummond PD. A review of peripheral biomarkers in major depression: the potential of inflammatory and oxidative stress biomarkers. Prog Neuropsychopharmacol Biol Psychiatry. 2014;48:102-111.

4. Perlis RH. Betting on biomarkers. Am J Psychiatry. 2011;168(3): 234-236.

5. Fornaro M, Aguglia E, Dell'Osso L, Perugi G. Could the underestimation of bipolarity obstruct the search for novel antidepressant drugs? Expert Opin Pharmacother. 2011;12(18):2817-2831.

6. Lavoie J, Illiano P, Sotnikova TD, Gainetdinov RR, Beaulieu JM, Hébert $\mathrm{M}$. The electroretinogram as a biomarker of central dopamine and serotonin: potential relevance to psychiatric disorders. Biol Psychiatry. 2014;75(6):479-486.

7. Nutt DJ. The role of dopamine and norepinephrine in depression and antidepressant treatment. J Clin Psychiatry. 2006;67 Suppl 6:3-8.

8. Lavoie J, Maziade M, Hébert M. The brain through the retina: the flash electroretinogram as a tool to investigate psychiatric disorders. Prog Neuropsychopharmacol Biol Psychiatry. 2014;48:129-134.

9. Perossini M, Fornaro P. Electroretinographic effects induced in humans by psychopharmacologic agents. Doc Ophthalmol. 1990; 75(1):1-6.

10. Filip V, Balik J. Possible indication of dopaminergic blockade in man by electroretinography. Int Pharmacopsychiatry. 1978;13(3): $151-156$.

11. Fornaro P, Castrogiovanni P, Perossini M, Placidi GF, Cavallacci G. Electroretinography (ERG) as a tool of investigation in human psychopharmacology. Electroretinographic changes induced by a combination of carbi-dopa and levo-dopa. Acta Neurol (Napoli). 1980;2(4): 293-299.

12. Bubl E, Tebartz Van Elst L, Gondan M, Ebert D, Greenlee MW. Vision in depressive disorder. World J Biol Psychiatry. 2009;10(4 Pt 2): 377-384.

13. Bubl E, Kern E, Ebert D, Bach M, Tebartz van Elst L. Seeing gray when feeling blue? Depression can be measured in the eye of the diseased. Biol Psychiatry. 2010;68(2):205-208.

14. Fornaro P, Castrogiovanni P, Dell'Osso L, Perossini M, Placidi G, Tassi G. Electroretinography (ERG) as a tool of investigation on dopaminergic activity in man. ERG changes induced by perphenazine and bromocriptine. Res Commun Psychol Psychiatr Behav. 1984;9:307-323.

15. Fornaro M, Bandini F, Ogliastro C, et al. Electroretinographic assessment in major depressed patients receiving duloxetine: might differences between responders and non-responders indicate a differential biological background? J Affect Disord. 2011;135(1-3):154-159.

16. Fornaro P, Dell'Osso L, Perossini M, Placidi GF, Castrogiovanni P. Effects of nomifensine on dopaminergic transmission in healthy volunteers evaluated by means of the electroretinographic technique. Acta Neurol (Napoli). 1984;6(1):5-10.

17. Bartel P, Blom M, Robinson E, van der Meyden C, Sommers DK, Becker $P$. The effects of levodopa and haloperidol on flash and pattern ERGs and VEPs in normal humans. Doc Ophthalmol. 1990;76(1): 55-64.

18. Porciatti V, Alesci R, Bagnoli P, Signorini G, Raffaelli A. Serotonin depletion modifies the pigeon electroretinogram. Doc Ophthalmol. 1989; 72(1):93-100.

19. Lavoie MP, Lam RW, Bouchard G, et al. Evidence of a biological effect of light therapy on the retina of patients with seasonal affective disorder. Biol Psychiatry. 2009;66(3):253-258.

20. Zawilska JB. The role of dopamine in the regulation of melatonin biosynthesis in vertebrate retina. Acta Neurobiol Exp (Wars). 1994;54 Suppl:47-56. 
21. Lavoie J, Rosolen SG, Chalier C, Hébert M. Negative impact of melatonin ingestion on the photopic electroretinogram of dogs. Neurosci Lett. 2013;543:78-83.

22. Srinivasan V, De Berardis D, Shillcutt SD, Brzezinski A. Role of melatonin in mood disorders and the antidepressant effects of agomelatine. Expert Opin Investig Drugs. 2012;21(10):1503-1522.

23. Martínez-Campa C, González A, Mediavilla MD, et al. Melatonin inhibits aromatase promoter expression by regulating cyclooxygenases expression and activity in breast cancer cells. Br J Cancer. 2009; 101(9):1613-1619.

24. Fornaro M, Prestia D, Colicchio S, Perugi G. A systematic, updated review on the antidepressant agomelatine focusing on its melatonergic modulation. Curr Neuropharmacol. 2010;8(3):287-304.

25. Sanchez Barcelo EJ, Martinez-Campa C, Mediavilla D, Gonzalez A, Alonso-Gonzalez C, Cos S. Melatonin and melatonergic drugs as therapeutic agents: ramelteon and agomelatine, the two most promising melatonin receptor agonists. Recent Pat Endocr Metab Immune Drug Discov. 2007;1:142-151.

26. Koesters M, Guaiana G, Cipriani A, Becker T, Barbui C. Agomelatine efficacy and acceptability revisited: systematic review and meta-analysis of published and unpublished randomised trials. $\mathrm{Br} J$ Psychiatry. 2013;203:179-187.

27. Howland RH. Publication bias and outcome reporting bias: agomelatine as a case example. J Psychosoc Nurs Ment Health Serv. 2011 49(9):11-14.

28. Opal MD, Klenotich SC, Morais M, et al. Serotonin 2C receptor antagonists induce fast-onset antidepressant effects. Mol Psychiatry. 2013.

29. Pérez-León JA, Sarabia G, Miledi R, García-Alcocer G. Distribution of 5-hydroxytriptamine2C receptor mRNA in rat retina. Brain Res Mol Brain Res. 2004;125(1-2):140-142.

30. Millan MJ, Gobert A, Lejeune F, et al. The novel melatonin agonist agomelatine (S20098) is an antagonist at 5-hydroxytryptamine2C receptors, blockade of which enhances the activity of frontocortical dopaminergic and adrenergic pathways. J Pharmacol Exp Ther. 2003; 306(3):954-964.

31. Milanese M, Tardito D, Musazzi L, et al. Chronic treatment with agomelatine or venlafaxine reduces depolarization-evoked glutamate release from hippocampal synaptosomes. BMC Neurosci. 2013;14:75.

32. Tardito D, Milanese M, Bonifacino T, et al. Blockade of stress-induced increase of glutamate release in the rat prefrontal/frontal cortex by agomelatine involves synergy between melatonergic and 5-HT2C receptor-dependent pathways. BMC Neurosci. 2010;11:68.

33. Sohn CH, Lam RW. Update on the biology of seasonal affective disorder. CNS Spectr. 2005;10(8):635-646; quiz 1-14.

34. Lam RW, Beattie CW, Buchanan A, Mador JA. Electroretinography in seasonal affective disorder. Psychiatry Res. 1992;43(1):55-63.

35. Stockton RA, Slaughter MM. b-wave of the electroretinogram. A reflection of ON bipolar cell activity. J Gen Physiol. 1989;93(1):101-122.

36. Green DG, Kapousta-Bruneau NV. A dissection of the electroretinogram from the isolated rat retina with microelectrodes and drugs. Vis Neurosci. 1999;16(4):727-741.

37. Gagné AM, Lavoie J, Lavoie MP, Sasseville A, Charron MC, Hébert M. Assessing the impact of non-dilating the eye on full-field electroretinogram and standard flash response. Doc Ophthalmol. 2010;121(3):167-175.

38. Kramer SG. Dopamine: a retinal neurotransmitter. I. Retinal uptake, storage, and light-stimulated release of H3-dopamine in vivo. Invest Ophthalmol. 1971;10(6):438-452.

39. Cohen AI, Todd RD, Harmon S, O'Malley KL. Photoreceptors of mouse retinas possess D4 receptors coupled to adenylate cyclase. Proc Natl Acad Sci U S A. 1992;89(24):12093-12097.

40. Muresan Z, Besharse JC. D2-like dopamine receptors in amphibian retina: localization with fluorescent ligands. J Comp Neurol. 1993;331(2): 149-160.

41. Biedermann B, Fröhlich E, Grosche J, Wagner HJ, Reichenbach A. Mammalian Muller (glial) cells express functional D2 dopamine receptors. Neuroreport. 1995;6(4):609-612.
42. Nguyen-Legros J, Simon A, Caillé I, Bloch B. Immunocytochemical localization of dopamine D1 receptors in the retina of mammals. Vis Neurosci. 1997;14(3):545-551.

43. Witkovsky P. Dopamine and retinal function. Doc Ophthalmol. 2004;108(1):17-40.

44. Tosini G, Fukuhara C. The mammalian retina as a clock. Cell Tissue Res. 2002;309(1):119-126.

45. Zawilska JB, Nowak JZ. Dopamine D4-like receptors in vertebrate retina: does the retina offer a model for the D4-receptor analysis? Pol J Pharmacol. 1997;49(4):201-211.

46. Pozdeyev N, Tosini G, Li L, et al. Dopamine modulates diurnal and circadian rhythms of protein phosphorylation in photoreceptor cells of mouse retina. Eur J Neurosci. 2008;27(10):2691-2700.

47. Hawlina M, Jenkins HG, Ikeda H. Diurnal variations in the electroretinographic c-wave and retinal melatonin content in rats with inherited retinal dystrophy. Doc Ophthalmol. 1992;79(2):141-150.

48. Zawilska J, Nowak JZ. Regulation of melatonin biosynthesis in vertebrate retina: involvement of dopamine in the suppressive effects of light. Folia Histochem Cytobiol. 1991;29(1):3-13.

49. Jaber M, Robinson SW, Missale C, Caron MG. Dopamine receptors and brain function. Neuropharmacology. 1996;35(11):1503-1519.

50. Missale C, Nash SR, Robinson SW, Jaber M, Caron MG. Dopamine receptors: from structure to function. Physiol Rev. 1998;78(1): 189-225.

51. Vallone D, Picetti R, Borrelli E. Structure and function of dopamine receptors. Neurosci Biobehav Rev. 2000;24(1):125-132.

52. De Berardis D, Marini S, Fornaro M, et al. The melatonergic system in mood and anxiety disorders and the role of agomelatine: implications for clinical practice. Int J Mol Sci. 2013;14(6):12458-12483.

53. Fujieda H, Scher J, Hamadanizadeh SA, Wankiewicz E, Pang SF, Brown GM. Dopaminergic and GABAergic amacrine cells are direct targets of melatonin: immunocytochemical study of mt1 melatonin receptor in guinea pig retina. Vis Neurosci. 2000;17(1):63-70.

54. Scher J, Wankiewicz E, Brown GM, Fujieda H. MT(1) melatonin receptor in the human retina: expression and localization. Invest Ophthalmol Vis Sci. 2002;43(3):889-897.

55. Manglapus MK, Iuvone PM, Underwood H, Pierce ME, Barlow RB. Dopamine mediates circadian rhythms of rod-cone dominance in the Japanese quail retina. J Neurosci. 1999;19(10):4132-4141.

56. Lavoie J, Gagne AM, Lavoie MP, Sasseville A, Charron MC, Hebert M. Circadian variation in the electroretinogram and the presence of central melatonin. Doc Ophthalmol. 2010;120(3):265-272.

57. Emser W, Dechoux R, Weiland M, Wirz-Justice A. Melatonin decreases the amplitude of the b-wave of the human electroretinogram. Experientia. 1993;49(8):686-687.

58. Gagné AM, Danilenko KV, Rosolen SG, Hébert M. Impact of oral melatonin on the electroretinogram cone response. J Circadian Rhythms. 2009;7:14.

59. Solís-Chagoyán H, Mendoza-Vargas L, Fuentes-Pardo B. Melatonin modulates the ERG circadian rhythm in crayfish. Comp Biochem Physiol A Mol Integr Physiol. 2008;149(4):373-379.

60. WMA Declaration of Helsinki: Ethical Principles for Medical Research Involving Human Subjects. Sixth Revision. Helsinki: WMA (Word Medical Association); 2008.

61. Weleber RG. The effect of age on human cone and rod ganzfeld electroretinograms. Invest Ophthalmol Vis Sci. 1981;20(3):392-399.

62. Brûlé J, Lavoie MP, Casanova C, Lachapelle P, Hébert M. Evidence of a possible impact of the menstrual cycle on the reproducibility of scotopic ERGs in women. Doc Ophthalmol. 2007;114(3): $125-134$.

63. Schneider T, Zrenner E. Effects of D-1 and D-2 dopamine antagonists on ERG and optic nerve response of the cat. Exp Eye Res. 1991;52(4): 425-430.

64. Marmor MF, Fulton AB, Holder GE, Miyake Y, Brigell M, Bach M; International Society for Clinical Electrophysiology of Vision. ISCEV Standard for full-field clinical electroretinography (2008 update). Doc Ophthalmol. 2009;118(1):69-77. 
65. Sharpley AL, Rawlings NB, Brain S, McTavish SF, Cowen PJ. Does agomelatine block 5-HT2C receptors in humans? Psychopharmacology (Berl). 2011;213(2-3):653-655.

66. Chenu F, Shim S, El Mansari M, Blier P. Role of melatonin, serotonin $2 \mathrm{~B}$, and serotonin $2 \mathrm{C}$ receptors in modulating the firing activity of rat dopamine neurons. J Psychopharmacol. 2014;28(2):162-167.
67. Fornaro M. Beyond monoamines towards the development of novel antidepressants. Journal of Psychopathology. 2012;18:226-233.

\section{Publish your work in this journal}

Neuropsychiatric Disease and Treatment is an international, peerreviewed journal of clinical therapeutics and pharmacology focusing on concise rapid reporting of clinical or pre-clinical studies on a range of neuropsychiatric and neurological disorders. This journal is indexed on PubMed Central, the 'PsycINFO' database and CAS, and is the official journal of The International Neuropsychiatric Association (INA). The manuscript management system is completely online and includes a very quick and fair peer-review system, which is all easy to use. Visit http://www.dovepress.com/testimonials.php to read real quotes from published authors.

\footnotetext{
Submit your manuscript here: http://www.dovepress.com/neuropsychiatric-disease-and-treatment-journal
} 